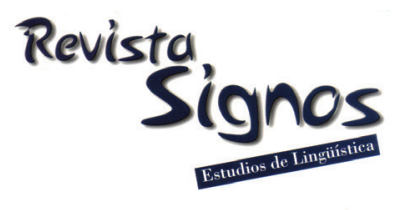

\title{
El préstamo en seis variedades geolectales del español: Un estudio en prensa escrita
}

\section{Loanwords in six geolectal varieties of Spanish: Research in written press}

\section{Constanza Gerding}

UNIVERSIDAD DE CONCEPCIÓN

Chile

cgerding@udec.cl
Mary Fuentes

Universidad de Concepción

Chile

marfuent@udec.cl

\section{Lilian Gómez}

UNIVERSIDAD DE CONCEPCIÓN

Chile

ligomez@udec.cl
Gabriela Kotz

Universidad de CONCEPCIÓN

Chile

gkotz@udec.cl

Recibido: 25-XI-2011 / Aceptado: 31-V-2012

\section{Resumen}

Con el propósito de constatar la vitalidad del español, en este estudio se realizó un análisis general de préstamos neológicos relevados de prensa escrita. El corpus estuvo constituido por préstamos con y sin adaptación clasificados en type y token frequencies por los nodos actualmente activos del Observatori de Neologia de la Universitat Pompeu Fabra entre 2003 y 2010. La recopilación de las unidades de estudio combinó la extracción manual con la captura semiautomática, y la clasificación se realizó de acuerdo al Protocolo de Vaciado de Prensa Escrita del Observatori. Se determinó la naturaleza de la adopción y la frecuencia de uso de los préstamos por informante, lengua de origen y área contextual del conocimiento. Además, se identificó rasgos diferenciadores del uso de algunos préstamos por informante y se analizó posibles motivaciones de los redactores de periódicos para incorporar préstamos al español. Se concluyó que los préstamos son un recurso importante de creación léxica, siendo los más frecuentes los anglicismos no adaptados.

Palabras Clave: Vitalidad lingüística, neologismo, préstamo, prensa escrita. 


\begin{abstract}
Seeking to probe the vitality of Spanish, a general analysis of neological loanwords found in the press was made. The corpus consisted of adapted and non-adapted loanwords, classified by type and token frequency between 2003 and 2010 by the six active nodes of the Observatori de Neologia of Universitat Pompen Fabra. Corpus collection combined manual and semiautomatic extraction, and unit classification was made according to standard protocol (Protocolo de Vaciado de Prensa Escrita del Observatori). It was possible to establish the nature of loanword adoption, frequency of use by informant, language of origin and contextual area of knowledge. Some distinctive features of use by informant and possible motivations for newspaper writers to incorporate loanwords into Spanish were also explored. It was concluded that loanwords are an important resource for lexical innovation, with non-adapted Anglicisms at the forefront.
\end{abstract}

Key Words: Language vitality, neologism, loanword, written press.

\title{
INTRODUCCIÓN
}

Las lenguas, como entes vivos que son, están sujetas a cambios. Una de las manifestaciones de estos cambios es la creatividad léxica, ya sea por mecanismos propios de una lengua (neologismos por derivación), por uso (variación sintáctica o semántica) o por la introducción de elementos ajenos a una lengua (préstamos). Si bien la influencia de unas lenguas en otras es una constante histórica, este fenómeno se ha acentuado en el mundo en el último tiempo con el avance de las comunicaciones y el mayor intercambio cultural.

De allí surgió la idea de examinar la vitalidad del español en la última década para verificar la fecundidad del préstamo como recurso de creación léxica. Así, el propósito de este trabajo fue describir los préstamos neológicos en prensa escrita recopilados por las Antenas Neológicas ${ }^{1}$. Para ello, se estimó pertinente determinar su frecuencia de uso por informante, lengua de origen y área del saber; clasificar las unidades según la naturaleza de la adopción y la frecuencia de aparición e identificar rasgos distintivos de algunos préstamos, y, por último, establecer comparaciones y buscar tendencias sobre el efecto de otras lenguas en el comportamiento neológico del español, la forma en que se importan voces y las posibles explicaciones de su incorporación a algunas variedades geolectales.

Las hipótesis de este trabajo planteaban que: a) la adopción es más frecuente que la adaptación entre los préstamos recogidos de la prensa escrita; b) los préstamos neológicos que recoge la prensa escrita son más frecuentes en determinadas áreas del conocimiento; c) de todos los préstamos neológicos, los anglicismos representan el mecanismo más frecuente de creación léxica; d) las lenguas amerindias aportan una proporción importante de préstamos a las bases de datos de las cinco Antenas 
latinoamericanas; y e) el catalán contribuye significativamente a la creación léxica del español de Barcelona.

\section{Marco referencial}

La multiplicidad de culturas y lenguas en contacto ha sido el resultado de conflictos bélicos, catástrofes naturales y expansión colonial; en consecuencia, la mayoría de las sociedades actuales se caracterizan por una diversidad de lenguas, etnias y culturas. 'Interculturalidad' e 'interculturalismo' destacan el diálogo y la conciencia positiva entre culturas diversas (Young, 1996; Benavides Ilizaliturri, 2005) y se asocian a la conducta humana en situaciones de interacción multicultural. Dicha conducta va a depender de las políticas de interculturalidad implícitas o explícitas que se adopten (Solís, 2006). Si bien las culturas se adaptan naturalmente al entorno a medida que se transmiten de una generación a otra, hoy la adaptación ocurre de modo más acelerado por efecto de las relaciones interculturales y la globalización, con sus costos y beneficios (Young, 1996; Huntington, 1997; Satori, 2001; Solís, 2006), como la asimilación de patrones de consumo y conducta impuestos por los medios (Benavides Ilizaliturri, 2005) y el riesgo de contaminación, desvirtuación o extinción de algunas culturas o lenguas minoritarias. Tal fenómeno es evidente en el lenguaje de los pueblos originarios, que luchan por rescatar sus raíces, salvaguardar sus tradiciones y preservar su identidad (Benavides Ilizaliturri, 2005). El contacto entre lenguas, catapultado por la globalización, se caracteriza por la tendencia al bilingüismo y la difusión de palabras y elementos gramaticales transmitidos de una lengua a otra (Appel \& Muysken, 2005), además de los elementos culturales denotados por estas.

Uno de los principales medios de desarrollo y expansión léxica que poseen las lenguas es el 'préstamo' (Delgado Álvarez, 2005; Esteban Asencio, 2008; Fuentes, Gerding, Pecchi, Kotz \& Cañete, 2009), término que se emplea para designar metafóricamente el trasvase (Gómez Capuz, 2009) o la cesión de elemento lingüísticos de un idioma a otro (Cardona 1991, en Lan, 2004). Préstamo también se refiere al proceso de transferencia, generalmente léxica, o al elemento lingüístico transferido (Gómez Capuz, 2009); es decir, no existen términos unívocos ni transparentes para definir estos fenómenos. Desde el punto de vista del contacto entre lenguas, Fontana y Vallduví (1990, en Márquez Rojas, 2006) hablan de 'alternancia de códigos', mientras que desde una perspectiva léxica y traductológica, se suelen utilizar los nombres de 'calco’ o ‘interferencia lingüística' (García Yebra, 1984). Gómez Capuz (2009), por su parte, establece una diferencia entre 'préstamo', que es la transferencia directa de significante y significado, y 'calco' léxico o estructural, que se refiere a la imitación de aspectos semánticos o morfológicos de una voz de la lengua de origen (skyscraper, en español 'rascacielos'). Otros autores hacen una distinción entre 'préstamo' y 'extranjerismo', reservando: 
"el nombre de préstamo para las voces de origen extranjero que han sido adaptadas al sistema fónico y morfológico de la lengua receptora y el de extranjerismo para las voces aceptadas tal como son en las lenguas de procedencia” (García Yebra, 1984: 333).

Lázaro Carreter (1968) plantea que el préstamo es un extranjerismo incorporado al sistema mediante mecanismos de adaptación, imitación o transformación. Abraham (1981, en Lan, 2004), a su vez, define el préstamo como un vocablo extranjero adaptado al sistema de la lengua receptora, mientras que el extranjerismo conserva la forma fónica y la acentuación primitivas. Gómez Capuz (2009), en cambio, plantea, con fines normativos, la dicotomía entre préstamo o préstamo adaptado y extranjerismo o préstamo no adaptado.

Otro término utilizado en este tipo de trasvase de léxico es el 'xenismo', que algunos autores definen como la asimilación gráfica de una voz extranjera, es decir, un préstamo adaptado que se emplea para referirse a personas, objetos, comidas, vestuario o ceremonias ajenos a la cultura receptora, aunque tenga adaptación fónica u ortográfica. Otros autores, sin embargo, utilizan criterios extralingüísticos y etnológicos para definir el xenismo como una realidad propia de una cultura extranjera (Gómez Capuz, 2009).

En consecuencia, existen diferentes términos para referirse a las voces que ingresan a una lengua desde otra. De hecho, en español coexisten 'préstamo', 'xenismo', 'voz extranjera', 'calco' y 'extranjerismo'. Ante tal diversidad conceptual y denominativa, en este trabajo se utilizará el término 'préstamo' para designar el vocablo que una lengua toma de otra, el que podrá ser una unidad adaptada o una no adaptada. 'Préstamo adaptado' será, entonces, el que sufra alguna modificación morfológica u ortográfica y 'préstamo no adaptado', el que visualmente se perciba idéntico a la unidad léxica de origen.

La prensa, terreno fértil para la creatividad léxica (Ortega Martín, 2001), refleja cambios como la revitalización de palabras, la formación de nuevos vocablos y el empleo de voces extranjeras. En efecto, el periodismo escrito es un vehículo de propagación de préstamos entre los hablantes del español (Diéguez, 2004; Delgado Álvarez, 2005; Sáez Godoy, 2005; Morin, 2006; Esteban Asencio, 2008; Fuentes, et al., 2009; Fuentes, Gerding, Pecchi, Kotz \& Cañete, 2010; Gerding, Fuentes \& Kotz, 2011). Además, los préstamos tienen carácter testimonial, porque dan cuenta de fenómenos sociales, económicos, políticos y culturales de una sociedad en una época determinada. Así, se observa la influencia de otras lenguas en variados ámbitos, aunque el uso de los préstamos se concentra en determinadas áreas y por motivos específicos. Por ejemplo, en la prensa tradicional o en la electrónica abundan los anglicismos en economía y comercio, computación y comunicación virtual, industria de la entretención, y deportes (Fuentes et al., 2010, Gerding et al., 2011). Las motivaciones, por su parte, pueden ser de diversa índole: economía lingüística, moda o vacío denominativo (Morin, 2006), propósito aclaratorio (Diéguez, 2004), impacto 
periodístico (López Castro, 2011), precisión (Esteban Asencio, 2008), atenuación, esnobismo y prestigio del hablante o del referente.

\section{Metodología}

No obstante que el Proyecto Antenas Neológicas recopila y estudia nuevas voces de distintos tipos de formación incorporadas al español, en este estudio la Antena Chilena de Neología aborda la presencia de préstamos con y sin adaptación, provenientes de diversas lenguas y repertoriados de prensa escrita entre el 1 de enero de 2003 y el 31 de diciembre de 2010 por los informantes que en la actualidad integran el Proyecto, a saber: Argentina, Barcelona, Chile, Colombia, México y Perú.

El corpus provino de las unidades neológicas recogidas en las lenguas que incluye el Protocolo de Vaciado de Prensa Escrita del Observatori de Neologia (2003): alemán, catalán, francés, griego, inglés, italiano, latín, ruso y vasco, además de la categoría 'Otros', que incluye las unidades que no pertenecen a ninguna de las lenguas individualizadas. Los préstamos neológicos fueron relevados de las siguientes fuentes:

\begin{tabular}{|l|l|l|}
\hline Informante & Periódicos & Ciudad de origen \\
\hline Argentina & Clarín, La Nación y Página/12 & Todos de la Ciudad de Buenos Aires \\
\hline Barcelona & El País y La Vanguardia & Barcelona y otras ciudades españolas \\
\hline Chile & El Mercurio y El Sur & Santiago y Concepción, respectivamente \\
\hline Colombia & El Tiempo y El Colombiano & Bogotá y Medellín, respectivamente \\
\hline México & La Jornada y El Universal & Ambos de Ciudad de México \\
\hline Perú & El Comercio, La República y Correo & Todos de Lima \\
\hline
\end{tabular}

Para una mejor contabilización de los datos, en este trabajo se presentan como un solo informante a las Antenas constituidas por más de un nodo: Barcelona y Colombia.

El procedimiento de selección y clasificación de neologismos combinó la extracción manual con la captura semiautomática mediante la herramienta 'Buscaneo' del Observatori de Neologia (OBNEO). Según el Protocolo de Vaciado de Prensa Escrita (2003), cada unidad neológica se registra una sola vez por periódico, por lo que la frecuencia de uso (token frequency) de las unidades recogidas podría redundar en una validez ecológica menor. Primero, se ingresa la unidad neológica a una ficha, se identifica y clasifica, y luego se somete a un proceso de corrección y validación, procedimiento con el que la unidad léxica ingresa al OBNEO en calidad de neologismo. En cada ficha neológica se describen las características principales de la nueva unidad: tipo de vaciado, entrada, contexto, tipo de unidad neológica, fuente, lengua de origen, ámbito geográfico de recolección, fecha de publicación y nota, en caso de requerirse una explicación especial (Fuentes et al., 2009).

La neologicidad de las unidades repertoriadas se determinó con criterio lexicográfico (Protocolo de Vaciado de Prensa Escrita, 2003; Adelstein \& Badaracco, 2004), es decir, mediante la verificación de su exclusión en los siguientes corpus de referencia: 
- Diccionario de la lengua española (Battaner, 2001)

- Diccionario de la lengua española (Real Academia Española, 2001)

- Diccionario de uso del español de América y España (Battaner, 2002)

Según este criterio, se descartaron como neologismos entradas como airbag, holding, reality show o ticket, por estar ya consignadas en uno o todos los corpus de exclusión.

Además, se identificaron las lenguas de mayor incidencia en el corpus, para lo cual se contabilizaron los préstamos con una token frequency de cinco unidades o más en cada lengua, se agruparon por tipo en 'adaptados' y 'no adaptados', y se clasificaron según las áreas contextuales del conocimiento en las que se presentaban con mayor frecuencia, de acuerdo a una adaptación de las áreas temáticas establecidas por CONICYT (2011).

\section{Resultados}

Del total de unidades neológicas repertoriadas $(\mathrm{N}=72.445)$, los préstamos correspondieron a 17.243 unidades. De esta manera y considerando la distinción token frequency/type frequency, el corpus estuvo constituido por $\mathrm{N}=17.243$ / 9.770 préstamos neológicos, de los cuales $\mathrm{N}=1.949$ / 1.238 fueron préstamos adaptados y $\mathrm{N}=15.294$ / 8.532, no adaptados. De dicho total, cerca de $22 \%$ correspondió a préstamos de diversas lenguas. Así, el corpus evidenció que los préstamos son uno de los recursos más abundantes para acuñar nuevas voces en español (Tabla 1), resultado que corrobora hallazgos de estudios anteriores (Esteban Ascencio, 2008; Fuentes et al., 2009; Fuentes et al., 2010).

Tabla 1. Frecuencias de aparición de neologismos y préstamos por informante.

\begin{tabular}{|r|c|c|c|c|}
\hline \multirow{2}{*}{ Informante } & \multicolumn{2}{|c|}{ Neologismos } & \multicolumn{2}{c|}{ Préstamos } \\
\cline { 2 - 5 } & Type frequency & Token frequency & Type frequency & Token frequency \\
\hline Argentina & 5.753 & 8.516 & 1.058 & 1.752 \\
\hline Barcelona & 19.609 & 32.154 & 4.747 & 8.711 \\
\hline Chile & 7.006 & 12.248 & 1.765 & 3.372 \\
\hline Colombia & 1.548 & 2.159 & 202 & 285 \\
\hline México & 2.701 & 4.180 & 411 & 543 \\
\hline Perú & 7.818 & 13.188 & 1.587 & 2.580 \\
\hline Totales & $\mathbf{4 4 . 4 3 5}$ & $\mathbf{7 2 . 4 4 5}$ & $\mathbf{9 . 7 7 0}$ & $\mathbf{1 7 . 2 4 3}$ \\
\hline
\end{tabular}

Como se observa en la Tabla 1, existe una relación directamente proporcional entre la cantidad de neologismos y las type y token frequencies de préstamos en todas las Antenas. También se observó gran variabilidad en la contribución de cada uno de los nodos respecto de los neologismos y, por lo tanto, también de los préstamos. A continuación, se presentan los principales análisis generales en torno a los préstamos: lengua de origen, tipo de unidad, frecuencia de aparición en la prensa y áreas temáticas en las que se presentaron. 
En relación con las lenguas de origen, la Tabla 2 ilustra la proporción de unidades en el corpus. Para este cálculo se consideraron las unidades de las cuatro primeras mayorías de préstamos en su token frequency por informante.

Tabla 2. Token frequency de préstamos en el corpus total, según lengua de origen.

\begin{tabular}{|r|c|c|}
\hline \multirow{2}{*}{ Lenguas } & \multicolumn{2}{|c|}{ Cantidad de préstamos (token) } \\
\cline { 2 - 3 } & Valores absolutos & Porcentajes \\
\hline Inglés & 11.912 & 74,26 \\
\hline Otros & 2.030 & 12,66 \\
\hline Francés & 1.030 & 6,42 \\
\hline Catalán & 745 & 4,64 \\
\hline Italiano & 257 & 1,60 \\
\hline Ruso & 42 & 0,26 \\
\hline Latín & 26 & 0,16 \\
\hline Total & $\mathbf{1 6 . 0 4 2}$ & $\mathbf{1 0 0 , 0 0}$ \\
\hline
\end{tabular}

El inglés se yergue como el idioma con la primera mayoría indiscutible de préstamos. En efecto, en todas las Antenas se evidenció un claro predominio de anglicismos no adaptados, lo que corrobora resultados de estudios anteriores (Fuentes et al., 2010, Gerding et al., 2011). La proliferación de anglicismos, que no es exclusiva del español, también ocurre, por ejemplo, en el alemán y el francés (Hagège, 1987). De hecho, el inglés es la lengua moderna que más préstamos ha provisto a otras por décadas (Moreno de Alba, 1992). En este estudio, el mayor número de anglicismos se concentró en actividades relacionadas con cuatro áreas principales: tecnologías de la información y computación (blogger, gamer, link); cultura y entretención (reggaetón, freak, rocanrol); deportes (esnórquel, nocaut, handbol), y economía, comercio y finanzas (subprime, retailer, forward). La tendencia de la prensa a preferir anglicismos obedece, por una parte, al predominio político, económico y cultural de Estados Unidos (Moreno de Alba, 1992) y a su influencia histórico-social y, por otra, a la urgencia de trasvasar al español mensajes originalmente redactados en inglés y la preferencia por emplear vocablos cortos, propia del estilo periodístico (Hagège, 1987).

El segundo lugar lo ocuparon los préstamos rotulados como 'Otros', categoría que aglutina un conjunto de lenguas, lo que le otorga un peso estadístico mayor y obliga a proceder con cautela respecto de su análisis. Muchos préstamos de 'Otros' correspondieron a xenismos referidos principalmente a cultura y entretención, con voces sobre vestuario, costumbres, normas, instrumentos musicales, bailes y ceremonias (burka, sharia, hawala, haram, mizraki, yeshiva, midrás, axé, cheiro, kultrún, trompe). Aunque las lenguas amerindias son propias de cinco de las Antenas, tuvieron escasa representación, siendo Chile el que presentó la menor cantidad $(<15 \%)$ de indigenismos (rehue, trompe, werkén), a diferencia de Perú (apu, hauylarsh, whipala), México (macábuitl, sendisho, chiquiguao), Argentina (qom-toba, buarpe, lonko) y Colombia (guipuñave, arhuaco, wivam). Las lenguas asiáticas aportaron neologismos esencialmente a la gastronomía (sushi, chifa, knaffe, wok, shitake). 
El tercer lugar lo ocupó el francés, principalmente con voces de la gastronomía (crutón, creme brulê) y la cultura y la entretención (déjà vu, pret-à-pôrter). Los préstamos del catalán, aportados esencialmente por Barcelona, figuraron en el cuarto lugar, en su mayoría asociados a las ciencias políticas y sociales, la cultura y la entretención, y el deporte (president, mosso, conselleria). En el quinto lugar se ubicó el italiano, con préstamos en la cultura y entretención (a capella, paparazzo) y la gastronomía (lasagna, risotto). Todos los nodos, salvo México, informaron de la presencia de algunos préstamos del ruso, lengua que se ubicó en el sexto lugar. Si bien el español históricamente ha tomado prestadas voces del latín, en este estudio su presencia fue muy baja en todas las Antenas $(<4 \%$ ), tal vez porque estas unidades son más esperables en textos científicos o medianamente especializados, mientras que en la prensa se tiende a banalizar la terminología (Cabré, 1993). En relación con las demás lenguas que contempla el Protocolo de Vaciado de Prensa Escrita (2003), se observaron escasos préstamos del alemán, el griego y el vasco en todas las Antenas, por lo que ninguno de estos idiomas se puede considerar estadísticamente relevante (Tablas 4 a 9).

Con respecto al tipo de préstamo, adaptado o no adaptado, también se determinaron las lenguas de mayor frecuencia por informante, según la token frequency (Tabla 3).

Tabla 3. Préstamos adaptados y no adaptados por lengua de procedencia e informante.

\begin{tabular}{|l|c|c|c|c|c|c|c|c|c|c|c|c|}
\hline \multirow{3}{*}{ Lenguas } & \multicolumn{10}{|c|}{ Informantes } \\
\cline { 2 - 16 } & \multicolumn{2}{|c|}{ Argentina } & \multicolumn{1}{|c|}{ Barcelona } & \multicolumn{2}{c|}{ Chile } & \multicolumn{2}{c|}{ Colombia } & \multicolumn{2}{|c|}{ México } & \multicolumn{2}{|c|}{ Perú } \\
\cline { 2 - 15 } & PA* & PNA** & PA & PNA & PA & PNA & PA & PNA & PA & PNA & PA & PNA \\
\hline Catalán & - & - & - & $3^{\circ}$ & - & - & - & - & - & - & - & - \\
\hline Francés & $4^{\circ}$ & $2^{\circ}$ & $3^{\circ}$ & $4^{\circ}$ & $3^{\circ}$ & $2^{\circ}$ & - & - & $3^{\circ}$ & $3^{\circ}$ & $4^{\circ}$ & $3^{\circ}$ \\
\hline Inglés & $1^{\circ}$ & $1^{\circ}$ & $1^{\circ}$ & $1^{\circ}$ & $1^{\circ}$ & $1^{\circ}$ & $1^{\circ}$ & $1^{\circ}$ & $2^{\circ}$ & $1^{\circ}$ & $1^{\circ}$ & $1^{\circ}$ \\
\hline Italiano & $3^{\circ}$ & $4^{\circ}$ & - & - & $4^{\circ}$ & $4^{\circ}$ & - & $3^{\circ}$ & $3^{\circ}$ & $4^{\circ}$ & $3^{\circ}$ & $4^{\circ}$ \\
\hline Latín & - & - & - & - & - & - & $3^{\circ}$ & $4^{\circ}$ & - & - & - & - \\
\hline Ruso & - & - & $4^{\circ}$ & - & - & - & $3^{\circ}$ & - & - & - & - & - \\
\hline Otros & $2^{\circ}$ & $3^{\circ}$ & $2^{\circ}$ & $2^{\circ}$ & $2^{\circ}$ & $3^{\circ}$ & $2^{\circ}$ & $2^{\circ}$ & $1^{\circ}$ & $2^{\circ}$ & $2^{\circ}$ & $2^{\circ}$ \\
\hline
\end{tabular}

$* \mathrm{PA}=$ préstamo adaptado $\quad * *$ PNA $=$ préstamo no adaptado

Como se observa en la Tabla 3, al separar los préstamos entre adaptados y no adaptados, en cuanto a la incidencia por lengua y por el lugar que estas ocupan, se repite la tendencia observada en la visión general (Tabla 2), pero hubo mayor dispersión y heterogeneidad en el tercer y cuarto lugar, donde alternaron el francés, el italiano, el latín y el ruso, con predominio de los galicismos en tercer lugar y de los italianismos, en cuarto. Esta diversidad se explicaría por la apertura hacia otras lenguas y culturas por parte de las distintas variedades del español estudiadas, especialmente en torno a la gastronomía y la entretención. La representación del alemán, el griego y el vasco fue escasa o nula. 
A continuación, se analizan los hallazgos más relevantes de cada Antena. Al igual que en los análisis anteriores, solo se consideró las cuatro categorías con las mayores frecuencias.

En Argentina, el primer lugar lo ocuparon los anglicismos, con predominio de las unidades no adaptadas (Tabla 4). Fernández (2001) atribuye la abundancia de anglicismos en la prensa argentina a la globalización de la economía y la política, junto con la evolución cíclica de las lenguas dominantes que, por razones de prestigio, llevarían a los hablantes cultos a aceptar voces anglicadas.

Tabla 4. Token y type frequencies aportadas por Argentina según lengua de procedencia.

\begin{tabular}{|l|c|c|c|c|}
\hline \multirow{2}{*}{ Lenguas } & \multicolumn{2}{|c|}{ Token frequency } & \multicolumn{2}{c|}{ Type frequency } \\
\cline { 2 - 5 } & $\begin{array}{c}\text { Préstamos } \\
\text { adaptados }\end{array}$ & $\begin{array}{c}\text { Préstamos } \\
\text { no adaptados }\end{array}$ & $\begin{array}{c}\text { Préstamos } \\
\text { adaptados }\end{array}$ & $\begin{array}{c}\text { Préstamos } \\
\text { no adaptados }\end{array}$ \\
\hline Francés & $\mathbf{1 9}(1,08 \%)$ & $\mathbf{9 1}(5,19 \%)$ & $\mathbf{1 9}(1,80 \%)$ & $\mathbf{6 5}(6,14 \%)$ \\
\hline Inglés & $\mathbf{9 1}(5,19 \%)$ & $\mathbf{1 . 3 4 8}(76,94 \%)$ & $\mathbf{7 2}(6,80 \%)$ & $\mathbf{7 4 7}(70,60 \%)$ \\
\hline Italiano & $\mathbf{2 1}(1,20 \%)$ & $\mathbf{4 7}(2,68 \%)$ & $\mathbf{1 8}(1,70 \%)$ & $\mathbf{1 8}(1,70 \%)$ \\
\hline Otros & $\mathbf{2 7}(1,54 \%)$ & $\mathbf{6 3}(3,60 \%)$ & $\mathbf{2 3}(2,17 \%)$ & $\mathbf{5 7}(5,39 \%)$ \\
\hline
\end{tabular}

Previsiblemente, el segundo lugar lo ocupó 'Otros', seguido del francés y del italiano. La influencia de estos últimos dos idiomas ha dejado rastro en el léxico argentino, principalmente en la gastronomía, aunque el francés es, además, marca de elegancia.

Barcelona recopiló la mayor cantidad de neologismos y, por ende, también de préstamos ('Tabla 5).

Tabla 5. Token y type frequencies aportadas por Barcelona según lengua de procedencia.

\begin{tabular}{|l|c|c|c|c|}
\hline \multirow{2}{*}{ Lenguas } & \multicolumn{2}{|c|}{ Token frequency } & \multicolumn{2}{c|}{ Type frequency } \\
\cline { 2 - 5 } & $\begin{array}{c}\text { Préstamos } \\
\text { adaptados }\end{array}$ & $\begin{array}{c}\text { Préstamos } \\
\text { no adaptados }\end{array}$ & $\begin{array}{c}\text { Préstamos } \\
\text { adaptados }\end{array}$ & $\begin{array}{c}\text { Préstamos } \\
\text { no adaptados }\end{array}$ \\
\hline Catalán & $\mathbf{3 0}(0,34 \%)$ & $\mathbf{7 4 5}(8,55 \%)$ & $\mathbf{2 5}(0,53 \%)$ & $\mathbf{3 4 1}(7,18 \%)$ \\
\hline Francés & $\mathbf{8 2}(0,94 \%)$ & $\mathbf{4 7 3}(5,43 \%)$ & $\mathbf{4 8}(1,01 \%)$ & $\mathbf{3 1 2}(6,57 \%)$ \\
\hline Inglés & $\mathbf{3 6 3}(4,16 \%)$ & $\mathbf{4 . 9 3 8}(56,70 \%)$ & $\mathbf{1 7 8}(3,75 \%)$ & $\mathbf{2 . 4 2 6}(51,11 \%)$ \\
\hline Otros & $\mathbf{3 0 5}(3,50 \%)$ & $\mathbf{8 1 3}(9,33 \%)$ & $\mathbf{1 7 9}(3,77 \%)$ & $\mathbf{6 1 4}(12,93 \%)$ \\
\hline
\end{tabular}

A diferencia de los demás informantes, Barcelona fue el único con presencia importante de préstamos en lengua catalana. Aun así, estos préstamos aparecieron en tercer y cuarto lugar, según la óptica de la observación: type o token frequency y adaptados o no adaptados, mientras que el primer lugar fue para los anglicismos, a excepción de la type frequency de préstamos adaptados, que correspondió a la categoría 'Otros', aunque con una diferencia mínima. Dadas la posición geográfica de Barcelona y su condición cosmopolita, los préstamos de la categoría 'Otros' fueron abundantes y de origen variado. Los galicismos destacaron en tercer lugar, los adaptados y, en cuarto, los no adaptados. 
La distribución de los préstamos de Chile es similar a la de Argentina (Tabla 6).

Tabla 6. Token y type frequencies aportadas por Chile según lengua de procedencia.

\begin{tabular}{|l|c|c|c|c|}
\hline \multirow{2}{*}{ Lenguas } & \multicolumn{2}{|c|}{ Token frequency } & \multicolumn{2}{c|}{ Type frequency } \\
\cline { 2 - 5 } & $\begin{array}{c}\text { Préstamos } \\
\text { adaptados }\end{array}$ & $\begin{array}{c}\text { Préstamos } \\
\text { no adaptados }\end{array}$ & $\begin{array}{c}\text { Préstamos } \\
\text { adaptados }\end{array}$ & $\begin{array}{c}\text { Préstamos } \\
\text { no adaptados }\end{array}$ \\
\hline Francés & $\mathbf{4 5}(1,33 \%)$ & $\mathbf{1 6 8}(4,98 \%)$ & $\mathbf{2 6}(1,47 \%)$ & $\mathbf{1 2 6}(7,14 \%)$ \\
\hline Inglés & $\mathbf{1 2 8}(3,79 \%)$ & $\mathbf{2 . 5 9 5}(76,97 \%)$ & $\mathbf{8 3}(4,70 \%)$ & $\mathbf{1 . 1 6 8}(66,17 \%)$ \\
\hline Italiano & $\mathbf{1 8}(0,53 \%)$ & $\mathbf{7 6}(2,25 \%)$ & $\mathbf{1 4}(0,79 \%)$ & $\mathbf{6 3}(3,57 \%)$ \\
\hline Otros & $\mathbf{1 0 6}(3,14 \%)$ & $\mathbf{1 3 7}(4,06 \%)$ & $\mathbf{8 6}(4,87 \%)$ & $\mathbf{1 1 4}(6,46 \%)$ \\
\hline
\end{tabular}

Al igual que en la mayoría de las Antenas, el primer lugar indiscutido lo ocuparon los anglicismos, con fuerte predominio de las unidades neológicas no adaptadas. A gran distancia le sigue la categoría 'Otros', con una leve ventaja de los no adaptados; se destaca aquí la presencia de xenismos de lenguas vernáculas y orientales. Las unidades neológicas del francés, que se presentaron en tercer lugar, correspondieron principalmente al ámbito de la gastronomía y a la actividad vitivinícola. Los préstamos del italiano ocuparon el cuarto lugar y, en general, respondieron a vacíos denominativos del área gastronómica.

Dado que el aporte de Colombia fue menor, es arriesgado realizar análisis generalizables. Es posible que la escasez de préstamos neológicos de esta base haya obedecido a la reciente incorporación de este nodo al Proyecto al momento de recopilar el material de este estudio. Con todo, su comportamiento fue similar al de las demás Antenas (Tabla 7).

Tabla 7. Token y type frequencies aportadas por Colombia según lengua de procedencia.

\begin{tabular}{|l|c|c|c|c|}
\hline \multirow{2}{*}{ Lenguas } & \multicolumn{2}{|c|}{ Token frequency } & \multicolumn{2}{c|}{ Type frequency } \\
\cline { 2 - 5 } & $\begin{array}{c}\text { Préstamos } \\
\text { adaptados }\end{array}$ & $\begin{array}{c}\text { Préstamos } \\
\text { no adaptados }\end{array}$ & $\begin{array}{c}\text { Préstamos } \\
\text { adaptados }\end{array}$ & $\begin{array}{c}\text { Préstamos } \\
\text { no adaptados }\end{array}$ \\
\hline Inglés & $\mathbf{3 2}(11,23 \%)$ & $\mathbf{1 8 2}(63,86 \%)$ & $\mathbf{2 2}(10,89 \%)$ & $\mathbf{1 1 8}(58,41 \%)$ \\
\hline Italiano & $\mathbf{1}(0,35 \%)$ & $\mathbf{6}(2,10 \%)$ & $\mathbf{1}(0,50 \%)$ & $\mathbf{5}(2,48 \%)$ \\
\hline Latín & $\mathbf{3}(1,05 \%)$ & $\mathbf{5}(1,75 \%)$ & $\mathbf{3}(1,48 \%)$ & $\mathbf{5}(2,48 \%)$ \\
\hline Otros & $\mathbf{2 3}(8,07 \%)$ & $\mathbf{2 3}(8,07 \%)$ & $\mathbf{1 8}(8,90 \%)$ & $\mathbf{2 1}(10,40 \%)$ \\
\hline
\end{tabular}

En efecto, la primera mayoría la ostentaron los anglicismos. En la categoría 'Otros', que ocupó el segundo lugar, también se advirtió la presencia de voces vernáculas, en especial, nombres de comunidades amerindias y vocablos orientales referidos a gastronomía.

Al igual que Colombia, México aportó una cantidad menor de préstamos en relación con las demás Antenas. Aun así, los préstamos no adaptados del inglés ocuparon el primer lugar (Tabla 8) y se referían mayormente a los deportes y a la 
información y la computación. Si bien México es un país geográficamente cercano a Estados Unidos, la incidencia de anglicismos fue levemente inferior a la de otros informantes, lo que tal vez no obedece a una política lingüística, sino a un sesgo en la redacción o en la recopilación.

Tabla 8. Token y type frequencies aportadas por México según lengua de procedencia.

\begin{tabular}{|l|c|c|c|c|}
\hline \multirow{2}{*}{ Lenguas } & \multicolumn{2}{|c|}{ Token frequency } & \multicolumn{2}{c|}{ Type frequency } \\
\cline { 2 - 5 } & $\begin{array}{c}\text { Préstamos } \\
\text { adaptados }\end{array}$ & $\begin{array}{c}\text { Préstamos no } \\
\text { adaptados }\end{array}$ & $\begin{array}{c}\text { Préstamos } \\
\text { adaptados }\end{array}$ & $\begin{array}{c}\text { Préstamos no } \\
\text { adaptados }\end{array}$ \\
\hline Francés & $\mathbf{8}(1,47 \%)$ & $\mathbf{1 5}(2,76 \%)$ & $\mathbf{7}(1,70 \%)$ & $\mathbf{1 4}(3,41 \%)$ \\
\hline Inglés & $\mathbf{6 1}(11,23 \%)$ & $\mathbf{3 3 9}(62,43 \%)$ & $\mathbf{3 5}(8,52 \%)$ & $\mathbf{2 4 3}(59,12 \%)$ \\
\hline Italiano & $\mathbf{8}(1,47 \%)$ & $\mathbf{1 0}(1,84 \%)$ & $\mathbf{6}(1,46 \%)$ & $\mathbf{9}(2,19 \%)$ \\
\hline Otros & $\mathbf{6 3}(11,60 \%)$ & $\mathbf{2 3}(4,24 \%)$ & $\mathbf{5 9}(14,36 \%)$ & $\mathbf{2 2}(5,35 \%)$ \\
\hline
\end{tabular}

Entre los préstamos con adaptación de México, el primer lugar lo ocupó 'Otros', con unidades procedentes de lenguas y culturas amerindias para denotar alimentos tradicionales y elementos propios de esas culturas. El segundo lugar lo ocupó alternadamente la categoría 'Otros' en préstamos no adaptados y los anglicismos, en adaptados.

La realidad de Perú fue similar a la de la mayoría de los informantes con respecto a la alta frecuencia de anglicismos adaptados y no adaptados, tanto en su type como en su token frequency (Tabla 9) y la mayoría de estas unidades tuvieron relación con economía y negocios, espectáculos y computación.

Tabla 9. Token y type frequencies aportadas por Perú según lengua de procedencia.

\begin{tabular}{|l|c|c|c|c|}
\hline \multirow{2}{*}{ Lenguas } & \multicolumn{2}{|c|}{ Token frequency } & \multicolumn{2}{|c|}{ Type frequency } \\
\cline { 2 - 5 } & $\begin{array}{c}\text { Préstamos } \\
\text { adaptados }\end{array}$ & $\begin{array}{c}\text { Préstamos no } \\
\text { adaptados }\end{array}$ & $\begin{array}{c}\text { Préstamos } \\
\text { adaptados }\end{array}$ & $\begin{array}{c}\text { Préstamos no } \\
\text { adaptados }\end{array}$ \\
\hline Francés & $\mathbf{2 5}(0,97 \%)$ & $\mathbf{1 0 4}(4,04 \%)$ & $\mathbf{2 3}(1,45 \%)$ & $\mathbf{8 4}(5,29 \%)$ \\
\hline Inglés & $\mathbf{2 1 6}(8,39 \%)$ & $\mathbf{1 . 6 1 9}(62,87 \%)$ & $\mathbf{8 5}(5,36 \%)$ & $\mathbf{9 2 9}(58,54 \%)$ \\
\hline Italiano & $\mathbf{2 9}(1,13 \%)$ & $\mathbf{6 0}(2,33 \%)$ & $\mathbf{1 2}(0,76 \%)$ & $\mathbf{4 1}(2,58 \%)$ \\
\hline Otros & $\mathbf{4 8}(1,86 \%)$ & $\mathbf{3 9 9}(15,50 \%)$ & $\mathbf{3 9}(2,46 \%)$ & $\mathbf{3 0 2}(19,03 \%)$ \\
\hline
\end{tabular}

La categoría 'Otros' ocupó el segundo lugar, debido a las lenguas vernáculas y asiáticas. El francés y el italiano figuraron en los dos siguientes lugares, tanto entre las unidades adaptadas como las no adaptadas en su type y token frequencies. Los italianismos se referían mayormente a gastronomía, deportes y espectáculos, y los galicismos, a moda, gastronomía y espectáculos.

A la luz de los resultados precedentes, cabe señalar que la proporción de préstamos que exhibe una lengua es variable, ya que los factores que intervienen en la incorporación de voces extranjeras no necesariamente coinciden de un país a otro (Langacker, 1973). 
Las principales motivaciones para introducir préstamos a través de la prensa serían: el contacto entre lenguas (Zamora, 1982), el prestigio lingüístico (Jingjing, 2004) y los vacíos denominativos (Abdel Raham, 1991). Este último fenómeno se aprecia en la alta frecuencia de anglicismos, por ejemplo, en deporte, computación, economía y comercio. De hecho, en los estudios de Diéguez (2004), Sáez Godoy (2005), Fuentes et al. $(2009,2010)$ y Gerding et al. (2011), se constató una fuerte presencia de anglicismos en la prensa chilena, la que reflejaría la disposición de esos hablantes a aceptarlos. En general, el predominio de anglicismos obedecería a la universalidad del inglés, por efecto del modelo de desarrollo social y económico en gran parte de América Latina, que impone junto con una lengua una dominación científico-técnica y cultural de las ideas (Hamel, 2004).

En muchos países latinoamericanos se realizan esfuerzos gubernamentales para fomentar el uso masivo del inglés a través de los medios de comunicación. Con esta política lingüística se pretende, por ejemplo, lograr que los egresados de enseñanza secundaria "tengan un nivel de inglés competitivo en el año 2019" (Ministerio de Educación de Colombia, 2011); de mantenerse esta política, podría aumentar la incidencia de anglicismos en el español.

La presencia de las lenguas vernáculas en la categoría 'Otros' fue disímil entre los informantes. En México, por ejemplo, existe una política lingüística que, desde 1992 "reconoce constitucionalmente la presencia de pueblos indígenas de cultura y lengua diversas" (Morris Bermúdez, 2007: 60), con el consiguiente derecho al uso, promoción y desarrollo de sus lenguas y culturas. Asimismo, fue en Perú, cuya población indígena representa casi un $40 \%$, donde se oficializó por primera vez una lengua indígena en América Latina (Godenzzi Alegre, 2001). Se puede entender, entonces, que las lenguas vernáculas tengan cierta presencia en la prensa de este país. En Colombia, en cambio, las políticas lingüísticas en favor de los pueblos amerindios y afrocolombianos aún son precarias, si bien la Constitución Política garantiza criterios de equidad con respecto al español (Etxebarria \& Trillos, 2002). Esta precariedad podría explicarse por la menor presencia $(3,4 \%)$ en la población colombiana de los más de ochenta pueblos indígenas, con más de 64 lenguas y 300 dialectos, según el censo de 2005 (Oróstegui Durán, 2008). Si bien la cultura de los pueblos originarios es evidente en algunos países, la escasez de voces nativas se podría deber a su desplazamiento geográfico, social y económico.

Por otra parte, se constató que el francés y el italiano eran relativamente importantes en el español de la mayoría de las latitudes estudiadas. El catalán, que solo fue significativo en Barcelona, es lengua oficial en Cataluña y el segundo idioma más usado luego del español (IDESCAT, 2011), probablemente gracias a políticas lingüísticas en su favor. En cuanto a la proximidad geográfica y el contacto entre lenguas, no se constató una influencia marcada de, por ejemplo, galicismos en Barcelona ni de anglicismos en México. 
A continuación se presenta un análisis de los préstamos adaptados y no adaptados más recurrentes recogidos por cada informante.

Tabla 1o. Préstamos con y sin adaptación con mayores token frequencies por informante.

\begin{tabular}{|c|c|c|c|c|c|}
\hline \multirow{2}{*}{ Antena } & \multirow{2}{*}{ Tipo } & \multicolumn{4}{|c|}{ Lugar de preponderancia } \\
\hline & & Primero & Segundo & Tercero & Cuarto \\
\hline \multirow{2}{*}{ Argentina } & PA* & biodiésel & burka & aggiornar & - \\
\hline & PNA** & blog / default & ballotage & pai & xeneize \\
\hline \multirow{2}{*}{ Barcelona } & PA & blogosfera & muyahidin & debú & kalashnikov \\
\hline & PNA & blog / online & canarinha & conseller en cap & déjà vu \\
\hline \multirow{2}{*}{ Chile } & $\mathrm{PA}$ & ránking & verdeamarelo & discotheque / tur & - \\
\hline & PNA & blog & terroir & - & risotto \\
\hline \multirow{2}{*}{ Colombia } & PA & reguetón & feng shui & - & - \\
\hline & PNA & blog & emberá & - & - \\
\hline \multirow{2}{*}{ México } & $\mathrm{PA}$ & buichol / kiliwa & - & discoteque & - \\
\hline & PNA & blog & - & champagne & - \\
\hline \multirow{2}{*}{ Perú } & $\mathrm{PA}$ & ránking & asháninka & panetón & ecran \\
\hline & PNA & blog & chakana & chiffon / femme fatale & figuretti \\
\hline
\end{tabular}

$*$ PA $=$ préstamo adaptado $\quad * *$ PNA $=$ préstamo no adaptado

Como se observa en la Tabla 10, el anglicismo no adaptado blog destaca por ser el préstamo más frecuente en todo el corpus. El uso preferente de blog frente a 'bitácora' en las TIC estaría dado por la brevedad del anglicismo y porque 'bitácora' se circunscribe al ámbito marino. Al igual que en otros niveles de análisis, los préstamos del inglés presentan la más alta frecuencia y en su mayoría corresponden a la computación. Por otra parte, llama la atención la recurrencia de xenismos, en especial de lenguas vernáculas, y la predominancia de galicismos, principalmente en gastronomía y entretención.

La riqueza del corpus refleja las motivaciones de hablantes y redactores de prensa para adoptar o adaptar préstamos, como se muestra en la Tabla 11, que presenta ejemplos de tal diversidad, organizados de acuerdo al posible motivo de incorporación. Cabe señalar que, si bien a veces existen equivalentes en español, persiste el uso de préstamos: game, nerd, online, ranking, sánduche, charme, beach volley, booklet, kerosene, fixture.

En ocasiones también se acompaña un extranjerismo con un equivalente en español a modo de explicación (Diéguez, 2004), tal vez como parte de un proceso de transición a la adopción o adaptación, lo que puede involucrar alternancia en la representación gráfica. Las motivaciones ejemplificadas en la Tabla 11 pueden ser lingüísticas, es decir, semánticas o morfológicas, cuando la vía de penetración es escrita, o fonéticas cuando es oral, o intralingüísticas, como ocurre con la motivación terminológica 
(Casas Gómez, 1996), y se materializan por distintos canales: agencias noticiosas, prensa periódica, industria y comercio, ciencias, cine y relaciones internacionales o sociales (Alfaro, 1970).

Uno de los préstamos interesantes de analizar fue el nombre con que se denomina 'complejo comercial': Argentina reportó mall en alternancia con shopping center y shopping, siendo este último más recurrente; en Barcelona, ambas voces tuvieron baja frecuencia y en Chile, al igual que en Perú, predominó mall frente a shopping o shopping center. En Colombia no se observaron dichos neologismos, pero en México coexistían, con baja frecuencia. Por otra parte, Barcelona Chile, Colombia y Perú reportaron, además, el uso de shopping como la 'actividad relativa a la adquisición de mercadería'.

Todos los nodos reportaron la variante ortográfica shopping y el plural shoppings, pero no el plural de mall; lo anterior demuestra la inestabilidad lingüística de los neologismos (Cabré, 1993). Finalmente, ningún informante reportó el uso de shopping mall o shopping plaza, unidades léxicas que dan origen a los préstamos referidos a 'centro comercial'. Nótese en este caso el aporte que el español hizo originalmente al inglés con la voz 'plaza', lo que demostraría la disposición al trasvase léxico de las lenguas.

Tabla 11. Posibles motivaciones para la adopción o adaptación: Préstamos ilustrativos.

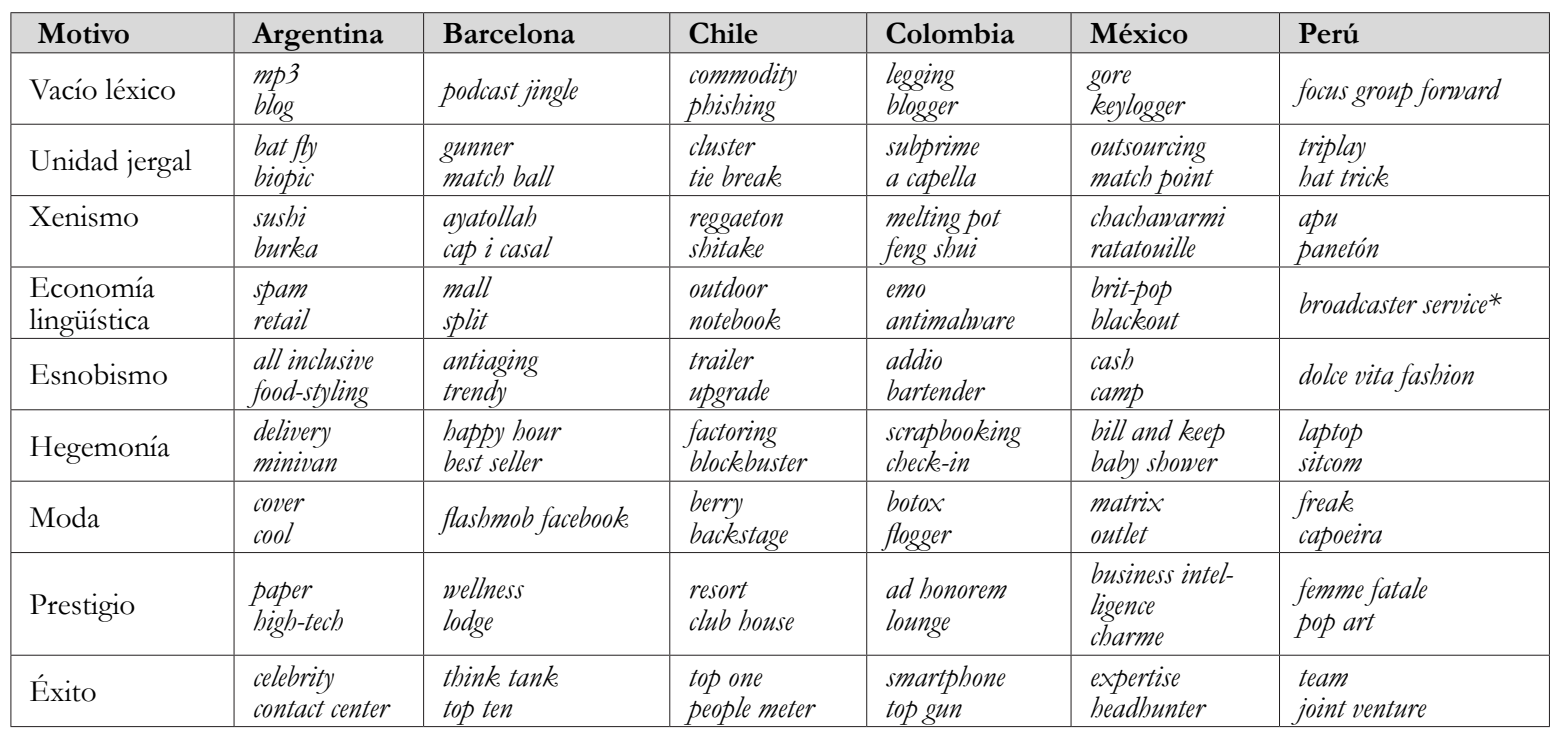

\footnotetext{
* Service se refiere aquí a 'subcontratador'.
} 
También resultó interesante de analizar la variabilidad del equivalente de 'computador(a)/ordenador portátil' según los distintos informantes. En Perú y Colombia figuró laptop en femenino, mientras que en México se recogió en ambos géneros. En Argentina, en cambio, coexistían laptop y notebook, ambos en femenino y, en Chile solo apareció notebook y únicamente en masculino. Barcelona no registró ninguno de los dos anglicismos. En cuanto al comportamiento del género gramatical en este caso, los hablantes probablemente mantienen el género original de 'ordenador', 'computador' o 'computadora' para sus símiles portátiles.

\section{CONCLUSIONES}

En términos globales, en este estudio se observó una relación directamente proporcional entre el total de unidades neológicas y los préstamos repertoriados, considerando la type y token frequencies y, por su incidencia, las voces extranjeras constituyeron un recurso muy prolífico de creación léxica.

En términos más específicos, se determinó que, en todas las Antenas, la mayor frecuencia de uso de préstamos neológicos correspondió a vocablos sin adaptación, siendo la inmensa mayoría voces provenientes del inglés. La categoría 'Otros' contribuyó con la segunda proporción más alta de préstamos; sin embargo, ninguna de ellas por separado constituyó un aporte significativo. El tercer lugar lo alternaron el francés y el italiano, dependiendo del informante. La categoría 'Otros' resulta compleja de clasificar y analizar, dada la variedad de lenguas y dialectos que dan origen a los préstamos englobados en ella y su disímil adaptación gráfica.

En cuanto a las áreas temáticas contextuales, un gran porcentaje de préstamos se concentró en los ámbitos de las tecnologías de la información y la computación, la cultura y la entretención, los deportes, la economía, el comercio y las finanzas, y la gastronomía.

En el análisis de algunos préstamos neológicos frecuentes, con presencia en todas las bases de datos, se constató que todos ellos correspondían a anglicismos, que alternaban en su forma adaptada y no adaptada y que algunos informantes tenían preferencia por una variante morfológica u ortográfica frente a otra.

Se pudo validar tres de las hipótesis de este estudio: a) los préstamos neológicos reflejan la influencia de las lenguas en determinadas áreas del saber, b) los anglicismos representan el mecanismo más frecuente de creación léxica en el corpus analizado, y c) la adopción es más frecuente que la adaptación entre los anglicismos recogidos.

En cuanto a las otras dos hipótesis, la evidencia demostró que, por una parte y contrario a lo esperado, las lenguas amerindias no contribuyen con una cantidad importante de préstamos al español de América Latina y, por otra, que la lengua catalana no realiza un aporte tan significativo a la creación léxica del español de Barcelona, como se había anticipado. 
Con respecto a las proyecciones de este estudio, la Antena Chilena de Neología asume como un desafío profundizar y expandir el abanico de investigaciones en relación con los préstamos lingüísticos. Por ejemplo, sería interesante realizar un análisis contrastivo de todas las bases de datos para buscar patrones comunes de comportamiento de los préstamos neológicos. Además, un sondeo entre redactores de prensa sobre el uso de los préstamos contribuiría a identificar sus motivaciones por determinadas preferencias. Finalmente, un estudio sobre disponibilidad léxica en lectores de prensa permitiría conocer el grado de transferencia de las lenguas en las áreas temáticas donde los préstamos son más abundantes. 


\section{REFERENCIAS BIBLIOGRÁFICAS}

Abdel Raham, W. H. (1991). A critical linguistic study of lexical borrowings in Arabic and English. Arts, 3(1), 33-36.

Adelstein, A. \& Badaracco, F. (2004). Teoría lingüística y estudios neológicos. Ponencia presentada en el Congreso Internacional Debates Actuales: Las Teorías Críticas de la Literatura y la Lingüística, Buenos Aires, Argentina.

Alfaro, R. (1970). Diccionario de anglicismos. Madrid: Gredos.

Appel, R. \& Muysken, P. (2005). Introduction: Bilingualism and language contact. En R. Appel \& P. Muysken (Eds.), Language contact and bilingualism (pp. 206-228). Amsterdam: Amsterdam University Press.

Benavides Ilizaliturri, L. (2005). Interculturalidad, globalización: Relación lengua, cultura $e$ identidades [en línea]. Disponible en: http://www.sabersinfin.com/index. php?option $=$ com_content\&task= view\&id $=61 \&$ Itemid $=89$

Battaner, M. P. (2001). Lema: Diccionario de la lengua española. Barcelona: Spes Editorial.

Battaner, M. P. (2002). Vox: Diccionario de uso del español de América y España. Barcelona: Spes Editorial.

Cabré, M. T. (1993). La terminología. Teoría, metodología y aplicaciones. Barcelona: Editorial Antártica/Empuries.

Casas Gómez, M. (1996). El poder mágico de la palabra. Trivium: Anuario de Estudios Humanísticos, 8, 29-52.

Clarín (2011). [En línea]. Disponible en: http://www.clarin.com/

Comisión Nacional de Investigación Científica y Tecnológica de Chile (CONICYT). (2011). [En línea]. Disponible en: http://www.conicyt.cl/573/channel.html

Correo (2011). [En línea]. Disponible en: http://diariocorreo.pe/

Delgado Álvarez, A. (2005). Los anglicismos en la prensa escrita costarricense. Káñina, 29 (Número Especial), 89-99.

Diéguez, M. I. (2004). El anglicismo léxico en el discurso económico de divulgación científica del español de Chile. Revista Onomázein, 10(2), 117-141.

El Colombiano (2011). [En línea]. Disponible en: http://www.elcolombiano.com

El Comercio (2011). [En línea]. Disponible en: http://elcomercio.pe/

El Mercurio (2011). [En línea]. Disponible en: http://diario.elmercurio.com/ 
El País (2011). [En línea]. Disponible en: http://www.elpais.com/global/

El Sur (2011). [En línea]. Disponible en: http://www.elsur.cl/

El Tiempo (2011). [En línea]. Disponible en: http://www.eltiempo.com/

El Universal (2011). [En línea]. Disponible en: http://www.eluniversal.com.mx/ noticias.htm

Esteban Asencio, L. (2008). Uso, origen y procesos de creación de neologismos en prensa española. Círculo de lingüistica aplicada a la comunicación, 33, 3-27.

Etxebarria, M. \& Trillos, M. (2002). Politica lingüistica y realidad sociolingüística en el Caribe Colombiano [en línea]. Disponible en: http://www.linguapax.org/congres/ Tallers/article1_esp.html

Fernández, F. J. (2001). Anglicismos en el español urbano de Salta [en línea]. Disponible en: http://www.ub.edu/filhis/culturele/salta.html

Fuentes, M., Gerding, C., Pecchi, A., Kotz, G. \& Cañete, P. (2009). Neología léxica: Reflejo de la vitalidad del español de Chile. RLA, Revista de Lingüïstica Teórica y Aplicada, 47(1), 103-124.

Fuentes, M., Gerding, C., Pecchi, A., Kotz, G. \& Cañete, P. (2010). Neológica léxica icorreo electrónico o e-mail? Ponencia presentada en el XII Simposio Iberoamericano de Terminología, RITerm, Buenos Aires, Argentina.

García Yebra, V. (1989). Teoría y práctica de la traducción. Madrid: Gredos.

Gerding, C., Fuentes, M. \& Kotz, G. (2011). Loanwords in the press: The influence of English in Chile. Ponencia presentada en el XIX World Congress Bridging Cultures, International Federation of Translators, San Francisco, California, USA.

Godenzzi Alegre, J. C. (2001). Política lingüistica y educación en el contexto latinoamericano: El caso del Perú. Ponencia presentada en el II Congreso Internacional de la Lengua Española, Instituto Cervantes, Valladolid, España.

Gómez Capuz, J. (2009). El tratamiento del préstamo lingüistico y el calco en los libros de texto de bacbillerato y en las obras divulgativas. Tonos, 17 [en línea]. Disponible en: http:// www.um.es/tonosdigital/znum17/subs/indice/IndiceTonos.htm

Hagège, C. (1987). Les français et les siècles. Paris: Éditions Odile Jacob.

Hamel, R. (2004). Las cuatro fronteras de la identidad lingüística del español: Lengua dominante $y$ dominada, lengua fronteriza y lengua internacional. Ponencia presentada en el III Congreso Internacional de la Lengua Española, Rosario, Argentina. 
Huntington, S. (1997). El choque de civilizaciones y la reconfiguración del orden mundial. Barcelona: Paidós.

Instituto de Estadísticas de Cataluña (IDESCAT). (2011). [En línea]. Disponible en: http://www.idescat.cat/es/

Jingjing, Y. (2004). A comparative study of the borrowings in English and Chinese. Tesis de magíster, Kyungpook National University, Kyungpook, Corea.

Lan, W. (2004). El anglicismo en el léxico chino mandarín y en el léxico español su incidencia en la enseñanza-aprendizaje de español como lengua extranjera. Tesis de doctorado, Universidad Complutense de Madrid, Madrid, España.

Langacker, R. W. (1973). Language and its structure: Some fundamental linguistic concepts. Nueva York: Harcourt Brace Jovanovich, Inc.

Lázaro Carreter, F. (1968). Diccionario de términos filológicos. Madrid: Gredos.

La Jornada (2011). [En línea]. Disponible en: www.jornada.unam.mx/ultimas/

La Nación (2011). [En línea]. Disponible en: http://www.lanacion.com.ar/

La República (2011). [En línea]. Disponible en: http://www.larepublica.pe/

La Vanguardia (2011). [En línea]. Disponible en: http://www.lavanguardia.com/

Página 12 (2011). [En línea]. Disponible en: http://www.pagina12.com.ar

López Castro, C. (2011). Neologismos y préstamos lingüísticos del español americano en la prensa y en la lengua italiana contemporánea [en línea]. Disponible en: http://cvc. cervantes.es/literatura/aispi/pdf/08/08_061.pdf

Márquez Rojas, M. (2006). Los anglicismos terminológicos integrales en los textos especializados del español. Estudios de Lingüistica Aplicada, 24(43), 11-29.

Ministerio de Educación de la República de Colombia (2011). Colombia promueve aprendizaje de inglés [en línea]. Disponible en: http://www.mineducacion.gov. $\mathrm{co} / \mathrm{cvn} / 1665 /$ article-115350.html

Moreno de Alba, J. G. (1992). Anglicismos léxicos en España y América. En Mamfre (Eds.), Diferencias léxicas entre España y América (pp. 195-230). Madrid: Mampfre.

Morin, R. (2006). Evidence in the Spanish language press of linguistic borrowings of computer and Internet-related terms. Spanish in Context, 3(2), 161-179. 
Morris Bermúdez, R. (2007). Al borde del multiculturalismo: Evaluación de la política lingüística del estado mexicano en torno a sus comunidades indígenas. CONfines, 3(5), 59-73.

Oróstegui Durán, S. (2008). Traducción de la Constitución Colombiana de 1991 a siete lenguas vernáculas. Reflexión Política, 10(19), 164-175.

Ortega Martín, M. P. (2001). Neología y prensa: Un binomio eficaz. Revista de Estudios Literarios Espéculo, 18, s/p.

Protocolo de Vaciado de Prensa Escrita (2003). Observatori de Neologia, Institut Universitari de Lingüística Aplicada, OBNEO, Universitat Pompeu Fabra. (Mimeo).

Real Academia Española de la Lengua (2001). Diccionario de la Lengua Española. Madrid: Espasa Calpe.

Sáez Godoy, L. (2005). Anglicismos en el español de Chile. Revista Atenea, 492, 171177.

Satori, G. (2001). La sociedad multiétnica. Pluralismo, multiculturalismo y extranjeros. Madrid: Santillana.

Solís, G. (2006). Interculturalidad: Encuentros y desencuentros en el Perú. Interculturalidad, 3 [en línea]. Disponible en: http://interculturalidad.org/numero03/2_01. htm\#ftn00

Young, R. (1996). Intercultural communication: Pragmatics, genealogy, deconstruction. Clevedon: Multilingual Maters Ltd.

Zamora, J. C. (1982). Amerindian loanwords in general and local varieties of American Spanish. Word, 33(1-2), 2, 159-169.

\section{NOTA}

1 El Proyecto Antenas Neológicas del Observatori de Neologia (OBNEO) es un conjunto de grupos de investigación liderado por el Institut Universitari de Lingüística Aplicada de la Universitat Pompeu Fabra de Barcelona, cuyo objetivo es analizar la aparición de neologismos en catalán y en español. 